\title{
The use of hydrophobic beads in triglyceride hydrolysis
}

\author{
Astri Nur Istyami ${ }^{1 *}$, Made Tri Ari Penia Kresnowati ${ }^{2}$, Tirto Prakoso ${ }^{3}$, Tatang Hernas Soerawidjaja ${ }^{4}$ \\ 1, 3,4 Department of Bioenergy and Chemurgy Engineering, Bandung Institute of Technology, Bandung, Indonesia \\ 2 Department of Food Engineering, Bandung Institute of Technology, Bandung, Indonesia
}

\section{Keywords \\ Fatty acid \\ Triglyceride hydrolysis \\ Hydrophobic beads \\ Polypropylene \\ Polyethylene \\ Polystyrene}

Received: 17 October 2017

Accepted: 16 January 2018

Published: 13 February 2018

\begin{abstract}
Fatty acid is an important intermediate substance in oleochemical industry. The demand of fatty acid is predicted to increase significantly in the future due to increasing demand of oleochemical products, specifically biofuel production through decarboxylation of fatty acids. For decades, fatty acid has been commercially produced via thermal hydrolysis of triglyceride, which requires high temperature and high pressure. Technology of fatty acid production in mild temperature and low energy consumption is currently developing through enzymatic triglyceride hydrolysis, which is also known as lipolysis. The economic feasibility of this technology is enhanced by immobilization of lipolytic enzymes, which frequently utilizes hydrophobic beads as immobilization supports. The aim of this research is to investigate the effect of hydrophobic beads on non-enzymatic hydrolysis of triglyceride. Experimental data suggest that higher conversion of triglyceride hydrolysis was attained with the use of several hydrophobic beads: polypropylene, polyethylene, and polystyrene. This phenomenon was not shown in utilization of hydrophilic materials, such as silica gel, silica stone, and rice bran acetone powder. It is hypothesized that the higher hydrolysis conversion is caused by mass transfer facilitated by hydrophobic beads, while in hydrophilic materials, water substrate was adsorbed and lowered the hydrolysis conversion. Experimental data show that performance of solids in triglyceride hydrolysis is not only affected by hydrophobicity, but also strongly affected by $\mathrm{pH}$ and surface area. Utilization of hydrophobic beads provides a low-cost and low-energy consumption to initiate triglyceride hydrolysis at room temperature, in the presence or the absence of lipolytic enzymes.
\end{abstract}

(C) 2018 The Author(s). Published by TAF Publishing.

\section{INTRODUCTION}

Sustainability is currently one of the most discussed industrial issues. As the global market shifts from unrenewable products to green products, oleochemical industry has started to take place of conventional petrochemical industry. Instead of processing fossil fuel, oleochemical industry converts plant oils and fats into products, such as surfactant, detergent, fuel, etc. The main component of plant oils and fats is triglyceride, which is processed into intermediate substances, i.e., fatty acid and fatty acid methyl ester. Fatty acid and fatty acid methyl ester are important commodities in plant oil producing countries, such as Indonesia, Malaysia, Canada, China, etc. Recent development shows that decarboxylation of fatty acid results in production of hydrocarbon [1]. Hydrocarbon produced from plant oils is known as 'drop-in biofuel', which is ready to be applied to current technology of transportation. The research opened novel potential of biofuel production technology. Following this development, it is predicted that the demand of fatty acid will increase dramatically. Fatty acid is produced through hydrolysis reaction of triglyceride described below:

$$
\text { Triglyceride }+3 \mathrm{H}_{2} \mathrm{O} \rightleftharpoons 3 \text { FattyAcids }+ \text { Glycerol }
$$

This reaction begins with bonding of hydroxide ion from $\mathrm{H} 2 \mathrm{O}$ with carbon atom from electrophile carboxylic group, conforming tetrahedral intermediate. The separating glycerol results in free fatty acids products. The current technology of fatty acid production is non-catalytic thermal hydrolysis, which is also known as Colgate-Emery process. This process operates at $250^{\circ} \mathrm{C}$ and 50 bar without catalyst,

\footnotetext{
${ }^{*}$ Corresponding author: Astri Nur Istyami

†email: anistyami@che.itb.ac.id
} 
and results in high product yield (more than 95\%) [2]. Although it requires huge amount of energy, this process is currently the most preferable option compared to its predecessor, such as Twitchell process, which requires organosulphate acid as catalyst [3], or soap acidification, which creates huge amount of waste. Colgate-Emery process requires minimum separation step and waste processing compared to both processes. Nevertheless, this technology depends on high temperature, which causes several drawbacks, such as thermal degradation, isomerization, dehydration, polymerization, and color darkening of product $[4,5,6]$, especially on oils with high polyunsaturated fatty acids content. In the future, technology of triglyceride hydrolysis in milder condition is necessary to produce fatty acids in more economical, energy-efficient technology, and to avoid undesired side reactions.

The key of high conversion in non-catalytic thermal hydrolysis is sufficient contact between triglyceride and water, which is facilitated by high temperature and high pressure. It is not thermodynamically feasible to reduce the temperature in triglyceride hydrolysis reaction [7] unless in the presence of catalyst. In this case, biocatalyst in the form of enzyme lipase has been studied abundantly due to its specific performance. Lipase has a unique interfacial activation, where the lid of active site opens in the presence of hydrophobic phase, facilitating contact of triglyceride and water [8]. The utilization of microbial lipase has been established for quite a long time, but in limited cases due to its high production cost [9].

Immobilization of lipases has been introduced to increase economic feasibility enzymatic process, especially when high-cost microbial lipase is utilized [9]. It minimizes product contamination by enzymes and allows lipase enzyme to be recovered and reused after reaction. Common methods of lipase immobilization are physical adsorption on supports, covalent bonding on supports, entrapment with resin matrix, and enzyme cross-linking. Among these methods, adsorption is the most frequently used due to simple procedure. Unlike covalent bonding or cross-linking, interaction of enzyme-support in physical adsorption method has low risk of enzyme denaturation. It is also reversible, enabling regeneration of immobilized lipase [10]. Physical adsorption immobilization involves van der Waals force, ionic interaction, and hydrogen bonding [11, 12]. Supports commonly used in adsorption methods can be hydrophobic, e.g., polypropylene [13] and octyl agarose [14], or hydrophilic, e.g., Celite [15], Kieselguhr, hydroxylapatite, alumina [16], and silica [17]. Hydrophobic supports are reported to increase lipolytic activity of immobilized lipase $[18,19]$ thus they are preferable than hydrophilic supports. Nevertheless, there has not been any single report concerning the effect of unattached supports on the conversion of triglyceride hydrolysis. To attain comprehensive understanding of immobilized lipase, it is necessary to analyze the behavior of supports in triglyceride hydrolysis process themselves. In this manuscript, we report the utilization of several beads and powder and their effects on triglyceride hydrolysis. We hypothesize that solid particles in reaction mixture will increase contact of triglyceride and water. The solid particles selected were beads and powder that is the potential for further application of enzymatic hydrolysis. Beads of polypropylene, polyethylene, and polystyrene were selected as representative of hydrophobic solids which has been claimed as excellent immobilization support of lipase [13]. Rice bran acetone powder was selected because rice bran has been reported to possess lipolytic activity [20], which is enhanced when the rice bran is pretreated with cold acetone [21]. Silica gel and silica stone were also observed in comparison to hydrophobic beads. Solid macroparticles are easy to be separated at the end of reaction, making them a practical support of lipase immobilization.

\section{MATERIAL AND METHODS}

\section{A. Material}

The hydrolysis was performed with commercial food grade refined palm oil (Bimoli, produced by PT Salim Ivomas Pratama Tbk). Commercial polypropylene beads, polyethylene beads, and polystyrene beads were provided by PT Chandra Asri, Tbk. Rice bran acetone powder was prepared through immersion of rice bran in cold acetone, which was then filtrated and dried at room temperature. Phosphate buffer ( $\mathrm{pH}$ 7.00) and ammonia- $\mathrm{NH}_{4} \mathrm{Cl}$ buffer (pH 8.25) were made with $20 \mathrm{mM}$ aqueous solution.

\section{B. Hydrolysis Reaction}

Hydrolysis reactions with solids were conducted by adding $0.50 \mathrm{~g}$ of solid beads or powder into $25.00 \mathrm{~g}$ of palm oil. Buffer solution ( $1 \mathrm{~mL} / \mathrm{mg}$ palm oil) was added and the mixture was agitated with magnetic stirrer (1000 rpm) for 6 hours. The agitation was then stopped, and after the product was settled for ca. 10 minutes, degree of hydrolysis of the thick upper layer of product was then determined.

In reaction component/ $t$ analysis, hydrolysis reaction was also conducted without the presence of solids to analyze the behavior of agitated oil and agitated oil-buffer mixture in the $6^{\text {th }}$ hour. These experiments were also conducted in 10 seconds to determine initial condition of each run. 


\section{Degree of Hydrolysis Determination}

Sample $( \pm 1.0 \mathrm{~g})$ was pipetted from upper layer of hydrolysis product into a solution of ethanol-chloroform solution (1:1 volumetric ratio) that had been neutralized with alcoholic $\mathrm{KOH}$. The mixture was gently shaken until the greasy sample completely solubilized in ethanol-chloroform solu- tion. Three drops of phenolphtalein (1\% alcoholic solution) indicator were then added. The degree of hydrolysis was determined by titration of sample with alcoholic $\mathrm{KOH}$ $0.1 \mathrm{~N}$. Titration was terminated when clear solution color turned into slightly pink, indicating the achievement of neutral point. Degree of hydrolysis was calculated using Equation 2 [22].

$$
\text { Degreeofhydrolysis }(\%)=\frac{V_{K O H} \times 10^{-1} \times M_{K O H} \times M M}{W t \times f} \times 100
$$

$V_{K O H}$ is the volume of Potassium Hydroxide Solution $(\mathrm{KOH})$ required $(\mathrm{mL})$ to neutralize sample, $M_{K O H}$ is exact concentration of $\mathrm{KOH}(0.1 \mathrm{M}), \mathrm{MM}$ is average molecular mass of fatty acids for palm oil, Wt is the exact sample weight $(\mathrm{g})$, and $\mathrm{f}$ is oil mass fraction in reaction mixture. $\mathrm{KOH}$ concentration was exactly determined by weighing Potassium Hydrogen Phtalate (KHP) ( \pm 0.100 g) and applying it to demineralized water until it was completely dissolved. Three drops of phenolphtalein ( $1 \%$ alcoholic solution) indicator were then added. KHF aqueous solution was titrated with alcoholic $\mathrm{KOH}$ and the concentration was calculated using Equation 3.

$$
M_{K O H}=\frac{w_{K P H} \times 10^{3}}{V_{K O H} \times M r_{K H P}}
$$

$w_{K H P}$ is the exact weight of potassium hydrogen phtalate (g), $V_{K O H}$ is volume of alcoholic $\mathrm{KOH}$ required in titration $(\mathrm{mL})$, and $M r_{K H P}$ is the molecular mass of potassium hydrogen phtalate (204.2).

\section{Beads Surface Area Determination}

Beads surface area of polypropylene, polyethylene, polystyrene, silica gel, and silica gel was determined using Brunauer-Emmett-Teller (BET) surface area analysis. Nitrogen was used as analysis gas.

\section{RESULTS AND DISCUSSION}

Experiments were conducted at mild temperature, atmospheric pressure, and in the absence of lipolytic enzymes. Thus, the process was focused on the occurring of triglyceride hydrolysis reaction with assistance of hydrophobic and hydrophilic solids.

Comparison between polypropylene beads, polyethylene beads, polystyrene beads, silica gel, silica stone, and rice bran acetone powder was observed in neutral and basic conditions. Buffer was used to maintain consistency of $\mathrm{pH}$ during hydrolysis reaction that produces fatty acids. Figure 1 and Figure 2 show hydrolysis results with buffer $\mathrm{pH}$ 7.00 and $\mathrm{pH} 8.25$, respectively. In the presence of buffer $\mathrm{pH}$
7.00 , the hydrolysis reaction was only slightly occurred, and there was no significant difference between the solids' performance. On the other hand, in the presence of buffer $\mathrm{pH}$ 8.25, disparity of conversion was shown by utilization of various solids in reaction mixture. It suggests that solids' assistance in triglyceride hydrolysis was dependent on $\mathrm{pH}$. Basic buffers are commonly chosen in enzymatic triglyceride hydrolysis due to the enzymes' optimum activity in this condition [23]. In this case, even in the absence of lipolytic enzymes, basic condition promotes higher conversion of triglyceride hydrolysis. It suggests that basic buffer provides enough hydroxyl group, which was needed to initiate the reaction mechanism. Higher $\mathrm{pH}$ might promote higher degree of hydrolysis, but in the cases where lipase enzyme is utilized, it is undesired to use buffer with $\mathrm{pH}$ above 8.5 , because most of the lipases are optimum at $\mathrm{pH}$ 7.0-8.5 [23]. Neutral condition shows almost no differences between the varied solids' performance, thus the following discussion will be focused on each solid's performance at pH 8.25.

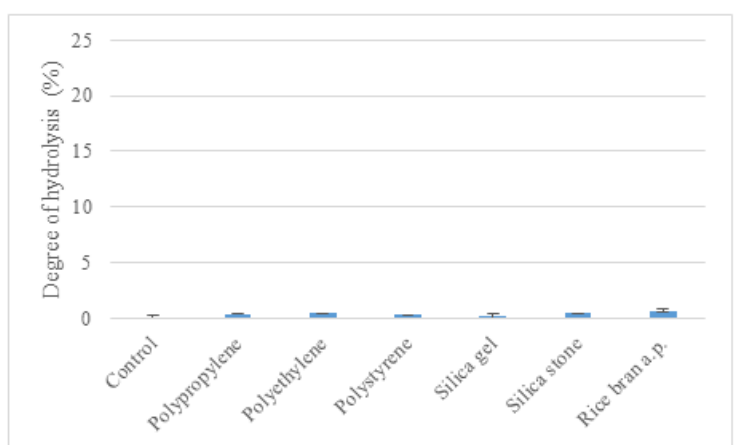

Fig. 1. Profile of palm oil hydrolysis using solid particles with assistance of buffer with $\mathrm{pH} 7.00$

From the experimental data, hydrophobic beads (polypropylene, polyethylene, and polystyrene) seem to increase conversion of hydrolysis compared to hydrolysis without beads. Utilization of hydrophobic beads in triglyceride hydrolysis has been acknowledged when lipolytic en- 
zymes are involved, e.g., when the beads are used as immobilization supports via adsorption method. Hydrophobic surface promotes interfacial activation in a similar way to lipolysis mechanism when lipase is contacted with oil substrates, enhancing lipolytic activity [13, 24].

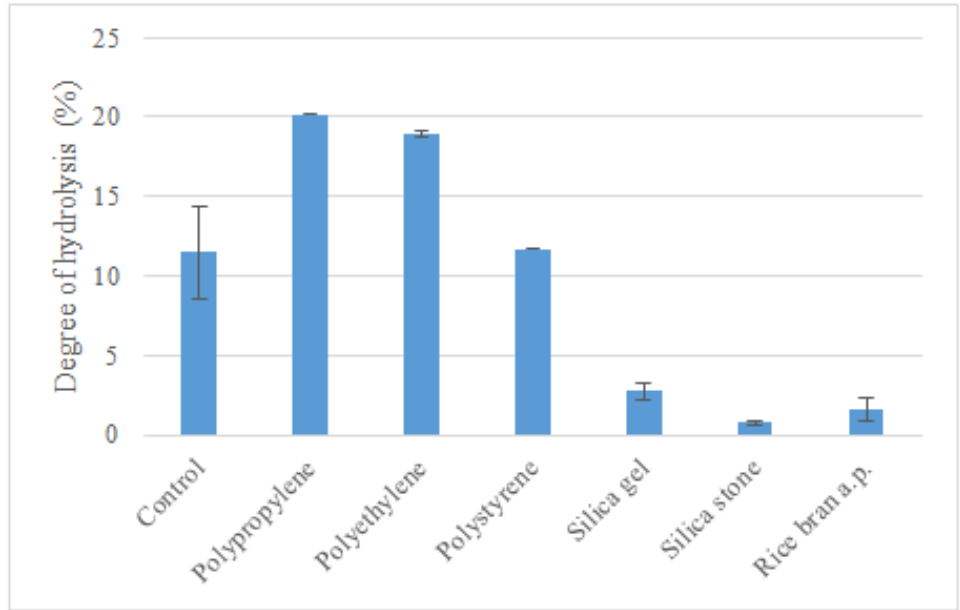

Fig. 2. Profile of palm oil hydrolysis using solid particles with assistance of buffer with pH 8.25

Interaction between lipase and hydrophobic surface changed lipase conformation into opened form, making it easier to be accessed [18], and hydrophobic surfaces around lipase active sites strongly adsorbed to the other hydrophobic surfaces [25]. Lipase adsorption onto hydrophobic solids was also claimed to be a simultaneous process of lipase purification and immobilization [13]. In this experiment, it was revealed that the hydrophobic beads themselves possess the ability to promote triglyceride hydrolysis. In the absence of lipolytic enzymes, beads agitated in reaction mixture increase collision between triglyceride and water molecules, facilitating more rapid mass transfer of substrates than in reaction mixture without beads. Sufficient contact is the key to high conversion in triglyceride hydrolysis. In the current commercial process of ColgateEmery, non-catalytic contact is facilitated by high temperature and pressure $\left(250^{\circ} \mathrm{C}, 50\right.$ bar). In Twitchell process, it is promoted by utilization of organosulphate acid. In enzymatic triglyceride hydrolysis, contact is facilitated by the active sites of lipase. The experiment result in this study suggests hydrophobic beads as low cost and low energy tools to initiate or to extend hydrolysis conversion of triglyceride to fatty acids. Separation of macromolecule beads is easier than powdered beads or enzyme molecule, ensuring high purity of fatty acids products.

On the other side, experiment result shows that utilization of hydrophilic solids (silica gel, silica stone, and rice bran acetone powder) reduces the degree of hydrolysis com- pared to hydrolysis without solids. The collision promoted by hydrophobic beads was not shown in the utilization of hydrophilic solids. It suggests that hydrophilic beads absorb water in mixture, reducing the molecules amount involved in reaction, holding the conversion due to equilibrium limitation. Even in the case that rice bran acetone powder is utilized, presence of rice bran lipase enzyme seems negligible due to the losses of water in mixture. It indicates the importance of hydrophobicity when solid particles are used in triglyceride hydrolysis.

For confirming reliability of the analysis described, reaction component analysis was conducted. Experiments were re-conducted in the absence of solids. Temperature, atmosphere, agitation speed, and reaction period were identical to the experiments with hydrophobic and hydrophilic solids. The results involving utilization of polypropylene beads, polyethylene beads, and rice bran acetone powder are shown in Figure 3, Figure 4, and Figure 5 respectively. Initial conditions of each process were analyzed by agitating oil or reaction mixture for 10 seconds. Reaction was terminated and acid value of the products was determined. All experiments in this condition showed zero conversion. Thus, it can be concluded that the hydrolysis reaction occurred during the agitation, and the source of acid value was not the plant oil, buffer, nor the solids themselves. In the $6^{\text {th }}$ hour of agitation of plant oil, no fatty acids were found. It confirms that hydrolysis of triglyceride requires sufficient water, which is not accomplished by only the humidity of 
reaction atmosphere. It also eliminates the possibility of degradation agent of triglyceride in the oil content.

Figure 3, Figure 4, and Figure 5 show that addition of $\mathrm{pH}$ 8.25 buffer into oil substrate increases fatty acid yield to a remarkable extent ( $>10 \%$ ). Basic condition provides enough hydroxyl group to initiate the reaction mechanism, as discussed above, even in the absence of beads. Combination of basic buffer and hydrophobic beads, i.e., polypropylene or polyethylene beads, increases the degree of hydrolysis to almost $20 \%$. Initiation of fatty acid formation is advantageous due to autocatalytic features in triglyceride hydrolysis [26], and it was provided low-cost and low-energy assistance of buffer and hydrophobic beads. This phenomenon was not shown in rice bran utilization. Although buffer increases the degree of hydrolysis to $11 \%$, addition of rice bran to buffer-oil mixture reduces the hydrolysis reaction. Hydrophilic character of rice bran decreases 'free water' in the mixture both in the form of original powder and acetone extract powder, suppressing the conversion of triglyceride hydrolysis.

Different performance in triglyceride hydrolysis occurs between the hydrophobic and hydrophilic beads themselves. To confirm the hypotheses that this performance related to mass transfer was facilitated by beads surface, the surface areas of several solids are shown in Table 1. Among hydrophobic beads that were utilized, polypropylene has the largest surface area, followed by polyethylene and polystyrene. This order is identical with the order of hydrophobic beads effect on the degree of hydrolysis (Figure 2). This phenomenon is also shown among the hydrophilic beads. We conclude that the surface area is an important variable involved in increasing degree of hydrolysis, because it facilitates contact between triglyceride and water molecules. Nevertheless, although silica gel and silica stone have the largest surface area among all of the beads observed, but their lack of hydrophobicity seems to inhibit the hydrolysis reaction. This shows dominancy of hydrophobicity effect compared to surface area effect on facilitating the hydrolysis reaction. Rice bran's hydrophilic character also inhibits the hydrolysis reaction, despite of the presence of lipase.

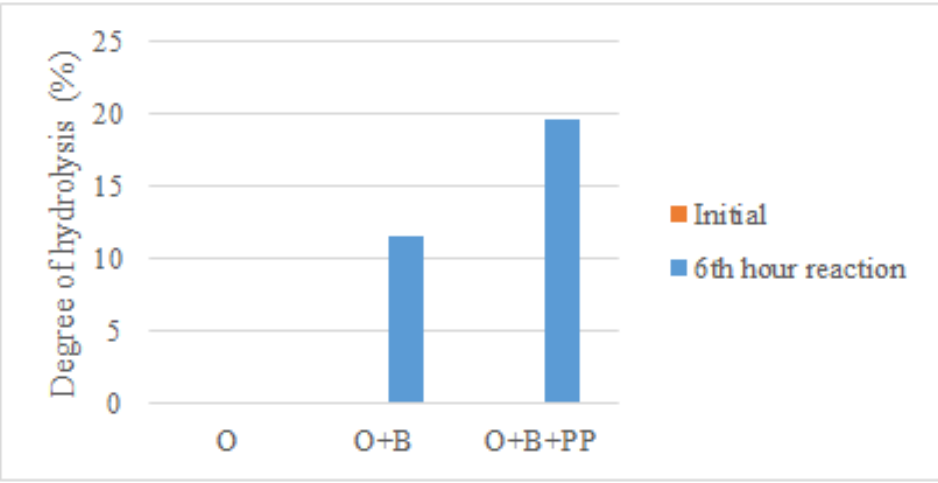

Fig. 3. Reaction component analysis of triglyceride hydrolysis involving polypropylene beads $(O=$ oil,$O+B=$ oil and buffer, $O+B+P P$ = oil, buffer, and polypropylene beads) 


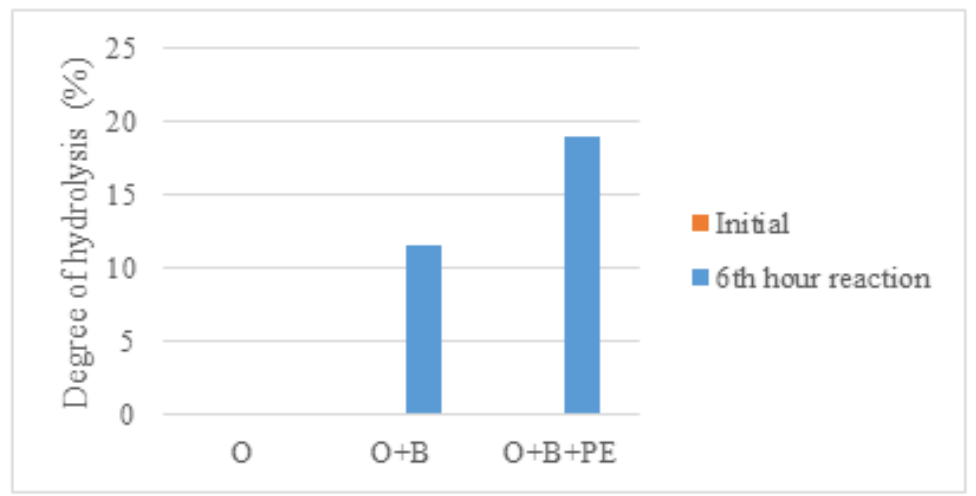

Fig. 4. Reaction component analysis of triglyceride hydrolysis involving polyethylene beads $(O=$ oil, $O+B=$ oil and buffer, $O+B+P P$ $=$ oil, buffer, and polyethylene beads)

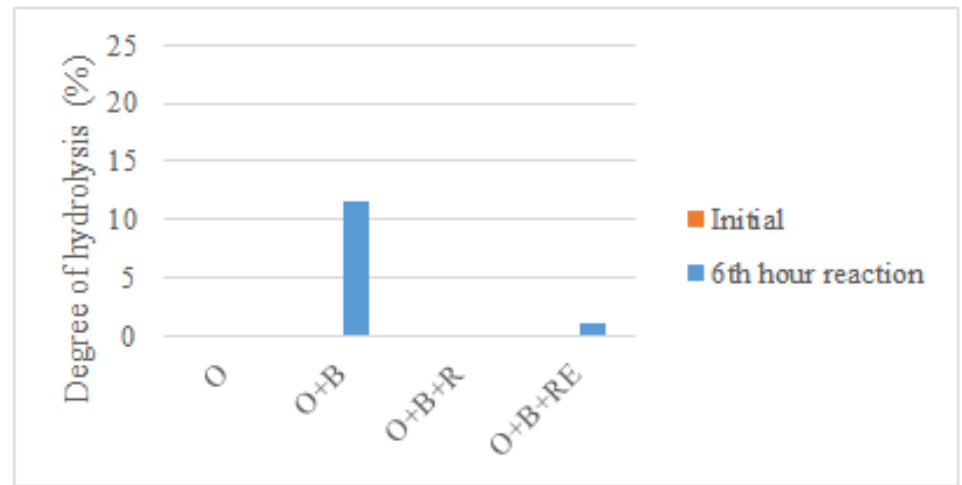

Fig. 5. Reaction component analysis of triglyceride hydrolysis involving rice bran powder $O=$ oil, $O+B=$ oil and buffer, $O+B+R=$ oil, buffer, and rice bran powder, $O+B+R E=$ oil, buffer, and rice bran acetone powder,)

TABLE 1

BET SURFACE AREA OF SOLIDS USED IN EXPERIMENT

\begin{tabular}{lc}
\hline \hline Beads Type & Surface Area $\left(\mathrm{m}^{2} / \mathrm{g}\right)$ \\
\hline Polypropylene & 110.005 \\
Polyethylene & 36.866 \\
Polystyrene & 1.815 \\
Silica gel & 263.882 \\
Silica stone & 186.002 \\
\hline \hline
\end{tabular}

In the recent development, lipase adsorption involving hydrophobic supports was reported to be a simple and efficient technique of lipase immobilization. It fixes the lid opening of lipase active sites, leading to higher lipolytic activity after immobilization [27]. Due to this condition, hydrophobic supports are claimed to be selective support for lipase immobilization [18]. Several hydrophobic supports have been reported as selective supports for lipase immobilization are polypropylene powder [13] and octyl agarose
$[18,27]$.

In our experiment, we reported that the hydrophobic solids themselves are useful to increase conversion in triglyceride hydrolysis, even when no lipase enzyme is impregnated. This method can be used to increase fatty acid content in reaction mixture. Triglyceride hydrolysis is an autocatalytic reaction, and the conversion is faster when sufficient fatty acid has been formed [26]. For attaining desirable yield, lipase can be immobilized with suitable support, preferably 
with high hydrophobicity and large surface area. Exploration of various hydrophobic solids is necessary to analyze the compatibility of lipase with supports, because in adsorption method, immobilization quality largely depends on interaction between lipase and supports. Larger surface area can be attained with smaller particle size, but extra fine powder might cause several troubles in the separation process. Optimization of this variable is necessary to be established before application. In the near future, the technology of low temperature triglyceride hydrolysis involving hydrophobic solids as lipase immobilization support is desired to attain competitive yield compared to the conventional thermal hydrolysis process.

\section{CONCLUSION}

The effect of hydrophobic and hydrophilic solids at low temperature triglyceride hydrolysis process has been reported in this experiment. Comparison between two different $\mathrm{pH}$ values suggests that basic condition is preferable in triglyceride hydrolysis, with or without beads. Under the absence of lipolytic enzymes, hydrophobic beads increased collision between triglyceride and water molecules, enhancing the degree of hydrolysis to almost 20\%. On the other hand, hydrophilic beads and powder decrease the degree of lipolysis, even when the surface area is large and lipase enzyme is contained. Hydrophilic character of solids inhibits hydrolysis reaction of triglyceride to almost zero conversion. It is concluded that hydrophobicity is an important factor in utilization of solids in triglyceride hydrolysis. In the case where hydrophobic solids are used, large surface area of the solids promotes higher conversion of hydrolysis. Several experiments were also conducted to confirm that enhancement of hydrolysis degree was triggered by the involvement of buffer and beads in the reaction, and the fatty acid product was not intrinsically contained in plant oil, buffer, or beads. This study results exposed that hydrophobic solids are not only useful in triglyceride hydrolysis when lipase enzyme is attached, but also in the absence of enzyme. Thus, it is recommended to be utilized in triglyceride hydrolysis, whether as a trigger to increase fatty acid content in reaction in the mixture or as lipase immobilization support. Compatibility of substance with lipase is important before determining the optimum particle size.

\section{REFERENCES}

[1] I. Kubičková, M. Snåre, K. Eränen, P. Mäki-Arvela, and D. Y. Murzin, "Hydrocarbons for diesel fuel via decarboxylation of vegetable oils," Catalysis To day, vol. 106, no. 1-4, pp. 197-200, 2005. doi: 10.1016/j.cattod.2005.07.188

[2] H. Barnebey and A. Brown, "Continuous fat splitting plants using the colgate-emery process," Journal of the American Oil Chemists' Society, vol. 25, no. 3, pp. 95-99, 1948. doi: 10.1007/bf02579733

[3] E. Twitchell, "Process of decomposing fats or oils into fatty acids and glycerin," US Patent 601 603, 1897.

[4] M. H. H. Hisham, A. A. Rahman, and I. S. Mustafa, "Uv-visible spectrophotometer analysis on polymerization of 2-hydroxyethyl methacrylate," International Journal of Applied and Physical Sciences, vol. 3, no. 2, pp. 42-45, 2017. doi: 10.20469/ijaps.3.50003-2

[5] N. Maneechot, W. Supiwong, and A. Tanomtong, "Chromosomal analysis and nors polymorphism of bagarius suchus (Siluriformes: Sisoridae) by conventional banding and fish techniques," Journal of Applied and Physical Sciences, vol. 2, no. 1, pp. 10-19, 2016. doi: 10.20474/-japs2.1.2

[6] R. W. M. Mounguengui, C. Brunschwig, B. Baréa, P. Villeneuve, and J. Blin, “Are plant lipases a promising alternative to catalyze transesterification for biodiesel production?" Progress in Energy and Combustion Science, vol. 39, no. 5, pp. 441-456, 2013. doi: 10.1016/j.pecs.2013.05.003

[7] A. N. Istyami, T. H. Soerawidjaja, and T. Prakoso, "Mass balances and thermodynamics study of thermal triglyceride hydrolysis," in 24th Conferences of Regional Symposium on Chemical Engineering, Semarang, Indonesia, 2017.

[8] H. Brockerhoff, "Action of pancreatic lipase on emulsions of water-soluble esters," Archives of Biochemistry and Biophysics, vol. 134, no. 2, pp. 366-371, 1969. doi: 10.1016/0003-9861(69)90295-1

[9] S. Seth, D. Chakravorty, V. K. Dubey, and S. Patra, "An insight into plant lipase research challenges encountered," Protein Expression and Purification, vol. 95, pp. 13-21, 2014. doi: 10.1016/j.pep.2013.11.006

[10] J. J. Virgen-Ortíz, S. G. Pedrero, L. Fernandez-Lopez, N. Lopez-Carrobles, B. C. Gorines, C. Otero, and R. FernandezLafuente, "Desorption of lipases immobilized on octyl-agarose beads and coated with ionic polymers after thermal inactivation. stronger adsorption of polymers/unfolded protein composites," Molecules, vol. 22, no. 1, pp. 91-100, 2017. doi: 10.3390/molecules22010091 
[11] T. Jesionowski, J. Zdarta, and B. Krajewska, "Enzyme immobilization by adsorption: A review," Adsorption, vol. 20, no. 5-6, pp. 801-821, 2014. doi: 10.1007/s10450-014-9623-y

[12] K. Rassiah, M. Ahmad, and A. Ali, "Effect on mechanical properties of rice husk/e-glass polypropylene hybrid composites using Sodium Hydroxide (NaOH)," Journal of Advances in Technology and Engineering Research, vol. 2, no. 4, pp. 105-112, 2016. doi: 10.20474/jater-2.4.1

[13] T. Gitlesen, M. Bauer, and P. Adlercreutz, "Adsorption of lipase on polypropylene powder," Biochimica et Biophysica Acta (BBA)-Lipids and Lipid Metabolism, vol. 1345, no. 2, pp. 188-196, 1997. doi: 10.1016/s0005-2760(96)00176-2

[14] E. A. Manoel, J. C. dos Santos, D. M. Freire, N. Rueda, and R. Fernandez-Lafuente, "Immobilization of lipases on hydrophobic supports involves the open form of the enzyme," Enzyme and Microbial Technology, vol. 71, pp. 53-57, 2015. doi: 10.1016/j.enzmictec.2015.02.001

[15] M. Würtz Christensen, L. Andersen, T. L. Husum, and O. Kirk, "Industrial lipase immobilization," European Journal of Lipid Science and Technology, vol. 105, no. 6, pp. 318-321, 2003. doi: 10.1201/b19219-4

[16] A. Macrae, "Lipase-catalyzed interesterification of oils and fats," Journal of the American Oil Chemists' Society, vol. 60, no. 2, pp. 291-294, 1983. doi: 10.1002/3527606033.ch11

[17] Y. Wan and D. Zhao, "On the controllable soft-templating approach to mesoporous silicates," Chemical Reviews, vol. 107, no. 7, pp. 2821-2860, 2007. doi: 10.1002/chin.200743247

[18] A. Bastida, P. Sabuquillo, P. Armisen, R. Fernandez-Lafuente, J. Huguet, J. M. Guisan et al., “A single step purification, immobilization, and hyperactivation of lipases via interfacial adsorption on strongly hydrophobic supports," Biotechnology and Bioengineering, vol. 58, no. 5, pp. 486-493, 1998. doi: 10.1002/(sici)1097-0290(19980605)58:5<486:: aid-bit4>3.0.co;2-9

[19] Q. Jin, G. Jia, Y. Zhang, Q. Yang, and C. Li, "Hydrophobic surface induced activation of pseudomonas cepacia lipase immobilized into mesoporous silica," Langmuir, vol. 27, no. 19, pp. 12 016-12 024, 2011. doi: 10.1021/la202794t

[20] J. R. Loeb, N. J. Morris, and F. Dollear, "Rice bran oil. iv. storage of the bran as it affects hydrolysis of the oil," Journal of the American Oil Chemists' Society, vol. 26, no. 12, pp. 738-743, 1949. doi: 10.1007/bf02903184

[21] A. N. Istyami, T. H. Soerawidjaja, T. Prakoso, and M. T. A. P. Kresnowati, "Effect of solvents on lipolytic activity of rice bran extract powder," in International Seminar on Chemical Engineering in Conjunction with Seminar Teknik Kimia Soehadi Reksowardojo, Bandung, Indonesia, 2016.

[22] D. Rooney and L. Weatherley, "The effect of reaction conditions upon lipase catalysed hydrolysis of high oleate sunflower oil in a stirred liquid liquid reactor," Process Biochemistry, vol. 36, no. 10, pp. 947-953, 2001. doi: 10.1016/ s0032-9592(01)00130-3

[23] M. Barros, L. Fleuri, and G. Macedo, "Seed lipases: Sources, applications and properties-a review," Brazilian Journal of Chemical Engineering, vol. 27, no. 1, pp. 15-29, 2010. doi: 10.1590/s0104-66322010000100002

[24] P. Adlercreutz, "Immobilisation and application of lipases in organic media," Chemical Society Reviews, vol. 42, no. 15, pp. 6406-6436, 2013. doi: 10.1039/c3cs35446f

[25] A. Brzozowski, U. Derewenda, Z. Derewenda, G. Dodson, D. Lawson, J. Turkenburg, F. Bjorkling, B. Huge-Jensen, S. Patkar, and L. Thim, "A model for interfacial activation in lipases from the structure of a fungal lipase-inhibitor complex," Nature, vol. 351, no. 6326, pp. 491-513, 1991. doi: 10.2210/pdb5tgl/pdb

[26] L. Lascaray, "Industrial fat splitting," Journal of the American Oil Chemists' Society, vol. 29, no. 9, pp. 362-366, 1952. doi: $10.1007 / \mathrm{bf02631459}$

[27] R. Fernandez-Lafuente, P. Armisén, P. Sabuquillo, G. Fernández-Lorente, and J. M. Guisán, "Immobilization of lipases by selective adsorption on hydrophobic supports," Chemistry and Physics of Lipids, vol. 93, no. 1, pp. 185-197, 1998. doi: 10.1007/978-1-59745-053-9_13 